\title{
UMA ESTRATÉGIA ADAPTADA PARA SÍNTESE DE MAGNETITA
}

\author{
Genilson Pereira Santana* e Alex Martins Ramos \\ Departamento de Química, Instituto de Ciências Exatas, Universidade Federal do Amazonas, Av. Gen. Rodrigo Octávio Jordão \\ Ramos, 3000, Campus Universitário, Coroado I, 69077-000 Manaus - AM, Brasil \\ José Domingos Fabris \\ Departamento de Química, Instituto de Ciências Exatas, Universidade Federal de Minas Gerais, Av. Antônio Carlos, 6627, \\ 31270-901 Belo Horizonte - MG, Brasil
}

Recebido em 3/1/07; aceito em 19/7/07; publicado na web em 19/12/07

\begin{abstract}
AN ADAPTED MAGNETITE SYNTHESIS STRATEGY. Chemical reactions in the solid state are often not included in undergraduate chemistry curricula, due to requirements for special laboratory facilities such as ovens and precision weighing balances. This work aims to describe novel and relatively simple magnetite chemical syntheses in dry medium, which could also be used as an alternative for freshmen chemistry experiments. The proposed reaction was carried out in a muffle furnace by heating (i) a sol-gel preparation and (ii) natural hematite, under nitrogen atmosphere at $400{ }^{\circ} \mathrm{C}$. The synthesized magnetite was characterized with powder $\mathrm{X}$-ray diffraction and Mössbauer spectroscopy. Results show that magnetite samples have chemical properties as well as crystalline structure quite similar to those of standard natural magnetite.
\end{abstract}

Keywords: iron oxides; Mössbauer spectroscopy; X-ray diffraction.

\section{INTRODUÇÃO}

Magnetita $\left(\mathrm{Fe}_{3} \mathrm{O}_{4}\right)$ é um óxido de ferro isoestrutural ao espinélio $\left(\mathrm{MgAl}_{2} \mathrm{O}_{4}\right)$, com distribuição catiônica invertida, em que os íons $\mathrm{Fe}^{3+}$ coordenam o oxigênio em simetria tetraédrica e $\mathrm{Fe}^{2+}$ e $\mathrm{Fe}^{3+}$ ocupam proporções equivalentes de sítios octaédricos. Estruturalmente, é representada por $\left[\mathrm{Fe}^{3+}\right]\left\{\mathrm{Fe}^{2+} \mathrm{Fe}^{3+}\right\} \mathrm{O}_{4}$, em que [ ] denota cátions nos sítios tetraédricos e \{\} nos octaédricos. A magnetita é muito estudada por sua importância econômica na indústria eletrônica (produção de cabeça reprodutora de som e vídeo, e de disquetes) e por ser um mineral acessório de inúmeras rochas, máficas, como basalto ${ }^{1,2}$, anfibolito $^{3}$ e tufito ${ }^{4,5}$, e não-máfica, como esteatito ${ }^{6,7}$ (pedra-sabão).

$\mathrm{Na}$ literatura, são relatados diversos caminhos de síntese de $\mathrm{Fe}_{3} \mathrm{O}_{4}$, como: hidrólise alcalina de solução de sulfato ferroso, para gerar $\mathrm{Fe}(\mathrm{OH})_{2}$, seguida por aquecimento do produto a $100{ }^{\circ} \mathrm{C}$ (reação de Schikorr $)^{8}$; redução de hematita a $400{ }^{\circ} \mathrm{C}$ em atmosfera de $5 \% \mathrm{H}_{2}$ e $95 \%$ de ar, saturada com vapor de água e livre de oxigênio ${ }^{9}$; transformação redutiva em uma ampola lacrada de uma suspensão de akaganeíta na presença de hidrazina em $\mathrm{pH}$ 9,5-11,5 e $100{ }^{\circ} \mathrm{C}^{10}$ e aquecimento de hidróxido acetato de ferro amorfo em atmosfera inerte de $\mathrm{N}_{2}{ }^{11}$.

Os caminhos apresentados acima possibilitam a síntese de magnetita, com diversos tamanhos e diferentes cristalinidades, sendo esta uma questão crucial na sua síntese, principalmente para o estudo da cinética de transformação. As magnetitas de pequeno tamanho formam primeiro maghemita $\left(\gamma-\mathrm{Fe}_{2} \mathrm{O}_{3}\right.$, um óxido de $\mathrm{Fe}^{3+}$, também isoestrutural ao espinélio), depois hematita $\left(\alpha-\mathrm{Fe}_{2} \mathrm{O}_{3}\right)^{12}$, em que as formas bem cristalizadas passam por magnetita não estequiométrica $\left(\mathrm{Fe}_{3-\mathrm{x}} \mathrm{O}_{4}\right)$ até $\mathrm{x}=0,33$, formando rapidamente $\gamma-\mathrm{Fe}_{2} \mathrm{O}_{3}$.

Outro fator que governa a estabilidade da magnetita é a presença de elementos traços. Nas magnetitas naturais é bastante comum a presença de $\mathrm{Cr}, \mathrm{Mg}$, Ti e $\mathrm{Mn}$, entre outros ${ }^{13,14}$. A presença de hetero-íons na estrutura da $\mathrm{Fe}_{3} \mathrm{O}_{4}$ faz com que a formação de $\gamma$ $\mathrm{Fe}_{2} \mathrm{O}_{3}$ se torne mais rápida e a de $\alpha-\mathrm{Fe}_{2} \mathrm{O}_{3}$, mais lenta ${ }^{15}$.

*e-mail: gsantana@ufam.edu.br
A análise de $\mathrm{Fe}_{3} \mathrm{O}_{4}$ por espectroscopia Mössbauer possibilita estudos de propriedades magnéticas, como transição Néel $\left(\mathrm{T}_{\mathrm{N}} \approx 858 \mathrm{~K}\right.$; temperatura de ordenamento magnético, no resfriamento) $)^{12}$, transição de Verwey $\left(\mathrm{T}_{\mathrm{v}} \approx 115 \mathrm{~K}\right)^{7,16,17}$ e fenômenos de relaxação superparamagnética ${ }^{18}$. A obtenção de uma amostra apropriada para determinado estudo é, assim, extremamente importante.

A difração de raios $\mathrm{X}$ pode fornecer informações sobre tamanho e estrutura dos cristais $^{19}$.

$\mathrm{O}$ presente trabalho propõe a síntese de $\mathrm{Fe}_{3} \mathrm{O}_{4}$, baseada na redução de íons $\mathrm{Fe}^{3+}$ em atmosfera de $\mathrm{N}_{2}$, utilizando um equipamento muito comum em laboratórios de Química (mufla) e um balão de destilação. Esses equipamentos proporcionam um experimento mais acessível economicamente (são de baixo custo) e são fáceis de manusear. Em seguida, o produto da reação será identificado por espectroscopia Mössbauer e por difração de raios X (método do pó).

\section{MATERIAIS E MÉTODOS}

Cerca de $231 \mathrm{~g}$ de $\mathrm{FeCl}_{3}$ anidro foram dissolvidos em $85 \mathrm{~mL}$ de água destilada e foram adicionados $100 \mathrm{~mL}$ de $\mathrm{NH}_{4} \mathrm{OH}$ concentrado, sob agitação à temperatura do ambiente. Após 15 min de vigorosa agitação, a mistura foi centrifugada (1800 rpm, $5 \mathrm{~min}$ ) e o gel lavado com solução de acetato de amônio (20\%). O gel foi secado em estufa a $80^{\circ} \mathrm{C}$, depois triturado em moinho de bolas, separado em peneira de 200 mesh e agitado mecanicamente durante $30 \mathrm{~min}$.

A literatura recomenda que os géis devam ser aquecidos em atmosfera inerte durante $2 \mathrm{~h}$ a $400{ }^{\circ} \mathrm{C}$, evitando com isso a oxidação da magnetita ${ }^{19}$. Um forno que permita essa separação pode ser relativamente caro. Foi, alternativamente, feita uma adaptação da técnica, utilizando-se uma mufla, um balão de vidro de $250 \mathrm{~mL}$ contendo uma saída alongada por um tubo de vidro, um balão elástico (do tipo de festa, tamanho grande), nitrogênio gasoso, padrão comercial, e dois tijolos refratários, com área suficiente para cobrir a entrada da mufla (Figura 1). O balão de vidro, contendo o material de síntese, é colocado dentro da mufla, de tal forma que o tubo de vidro atravesse o orifício do tijolo. O balão elástico, parcialmente 
cheio com nitrogênio, para manter a atmosfera inicialmente inerte, foi conectado ao tubo de vidro. O sistema retém gases orgânicos, liberados durante o aquecimento, para tornar a atmosfera progressivamente redutora (Figura 1).

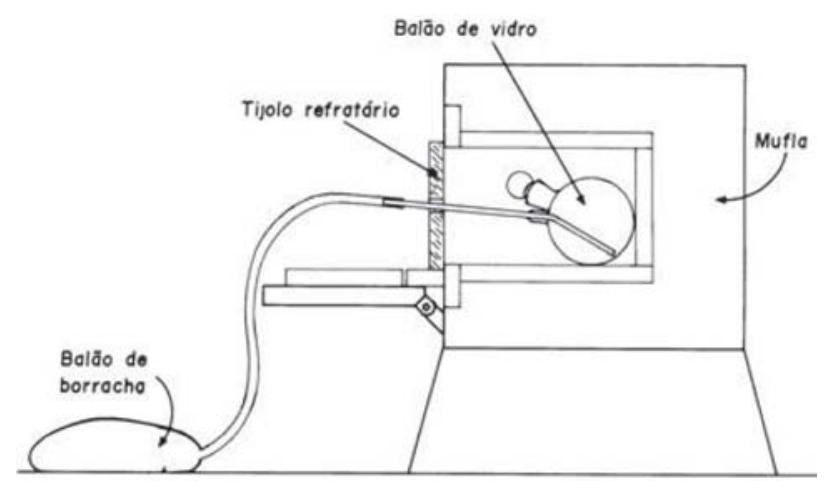

Figura 1. Montagem utilizada na síntese de magnetita

Foram sintetizadas também outras magnetitas, utilizando-se, com o substrato natural, a hematita, que foi coletada em uma mina localizada a cerca de $40 \mathrm{~km}$ de Belo Horizonte. Cinco gramas de uma amostra de minério de ferro contendo 74,0 massa\% de $\mathrm{Fe}_{2} \mathrm{O}_{3}$ foram colocadas dentro do balão de vidro. $\mathrm{O}$ mesmo procedimento de aquecimento para síntese da magnetita foi adotado para a hematita.

As magnetitas sintetizadas foram caracterizadas por difração de raios X, em difratômetro Rigaku modelo Geigerflex, com monocromador de grafite e radiação $\mathrm{Cu}\left(\mathrm{K}_{\alpha}\right)$, e por espectroscopia Mössbauer na temperatura do ambiente (aproximadamente, $298 \mathrm{~K}$ ), em espectrômetro com transdutor e gerador de função CMTE modelo MA250 e uma fonte de ${ }^{57} \mathrm{Co} / \mathrm{Rh}$, com atividade da ordem de $20 \mathrm{mCi}$.

\section{RESULTADOS E DISCUSSÃO}

Os difratogramas de raios X (Figura 2) mostram que a síntese das magnetitas ocorreu, nas duas rotas utilizadas: (i) pela redução da hematita e (ii) de um óxido de ferro amorfo produzido via sol-gel. A principal característica nos dois processos de síntese é a obtenção de um óxido bem cristalizado, indicado pela presença de reflexões com largura a meia-altura da mesma ordem do padrão interno $(\mathrm{NaCl})$ e da magnetita comercial (pura). Isso é confirmado pelos valores de parâmetro de rede das duas magnetitas sintetizadas, determinados pelo método de Nelson-Rilley ${ }^{19}$, que foram de $\mathrm{a}=0,8406(5) \mathrm{nm}$, para o cristal sintetizado via hematita, e a $=0,8401(5) \mathrm{nm}$, para a síntese via sol-gel. Esses valores são muito próximos do valor tabelado no "Joint Commitee on Powder Diffraction Standards (JCPDS)" que é $\mathrm{a}=0.8396(7) \mathrm{nm}$ (cartão n. 19-629).

Os espectros Mössbauer obtidos a 298 K (Figura 3) apresentam basicamente dois sextetos característicos de magnetita, conforme os parâmetros

Tabela 1. Parâmetros hiperfinos dos óxidos de ferro natural e sintetizando. $\mathrm{B}_{\mathrm{h}}=$ campo magnético hiperfino, $\delta=$ deslocamento isomérico e $\Delta=$ desdobramento quadrupolar

\begin{tabular}{lcccc}
\hline Rota Sintética & Sítio & $\mathrm{B}_{\mathrm{h}}(\mathrm{T})$ & $\delta\left(\mathrm{mm} \mathrm{s}^{-1}\right)$ & $\Delta\left(\mathrm{mm} \mathrm{s}^{-1}\right)$ \\
\hline Hematita & A & 47,0 & 0,69 & 0,01 \\
& B & 49,2 & 0,28 & $-0,01$ \\
Sol-gel & A & 46,9 & 0,67 & 0,02 \\
\multirow{3}{*}{ Magnetita natural } & B & 48,0 & 0,30 & $-0,01$ \\
& A & 46,0 & 0,68 & 0,01 \\
& B & 49,1 & 0,28 & $-0,01$ \\
\hline
\end{tabular}

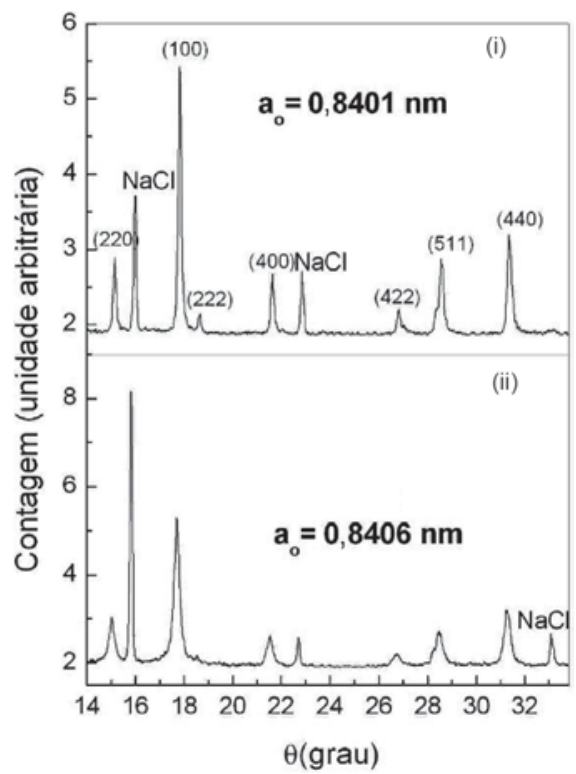

Figura 2. Difratogramas de raios $X$ para amostras de magnetita sintética, obtidas a partir ( $i$ ) da rota sintética sol-gel e (ii) da hematita, como precursor. $\mathrm{NaCl}=$ padrão interno

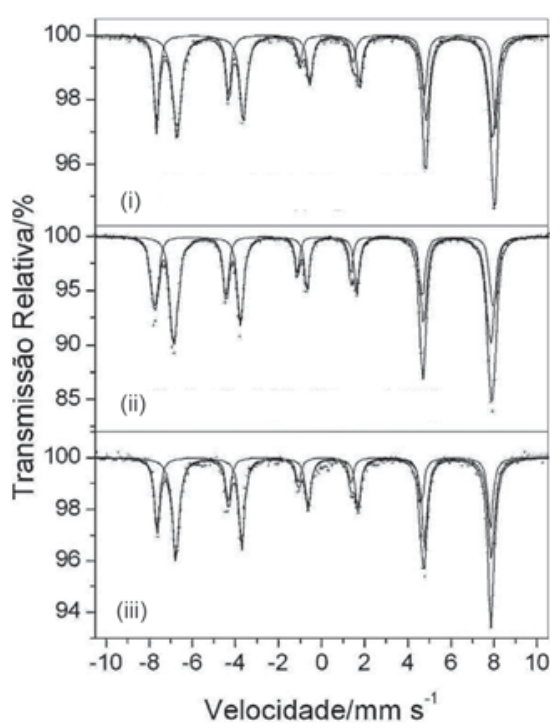

Figura 3. Espectros Mössbauer do ${ }^{57} \mathrm{Fe}$, a $298 \mathrm{~K}$, para amostras de magnetita (i) natural e sintéticas, obtidas a partir (ii) da rota sol-gel e a (iii) da hematita, como precursor

hiperfinos mostrados na Tabela 1. A razão entre área relativa ao sítio tetraédrico $\left(R_{A}\right)$ e sítio octaédrico $\left(R_{B}\right)$ foi determinada considerando-se que as linhas eram lorentzianas ${ }^{21}$. Os resultados foram $R_{B} / R_{A}=1,91$ (via hematita) e 1,89 (via sol-gel). Além disso, os espectros Mössbauer são caracterizados pela ausência de linha na região central de dubletos, típicas do fenômeno de relaxação superparamagnética, causada por partículas de pequenos tamanhos ${ }^{19,22}$. Estes resultados revelam que os dois caminhos de síntese levam à formação de uma magnetita estequiométrica, comparável a uma magnetita natural bem cristalizada, com baixo grau de substituição em cromo ${ }^{23}$.

\section{CONCLUSÕES}

Os dados mostram que a síntese de magnetita através da redução de $\mathrm{Fe}^{3+}$ leva à formação de um espinélio bem cristalizado e estequiométrico para as duas vias sintéticas. Fica ainda evidente 
que a adaptação da técnica leva uma síntese mais econômica, pois os materiais utilizados (tijolo refratário, balão de borracha comum e balão de destilação) são facilmente encontrados em laboratório, e, sobretudo, eficiente, pois os difratogramas de raios X (Figura 2), bem como os espectros Mössbauer (Figura 3) confirmam que a síntese via sol-gel e a partir de hematita levam a produtos com propriedades físicas e químicas características do óxido puro.

\section{REFERENNCIAS}

1. Jesus Filho, M. F.; Fabris, J. D.; Goulart, A. T.; Coey, J. M. D.; Ferreira, B. A.; Pinto, M. C. F.; Clay Clay Miner. 1995, 43, 641

2. Pinto, M. C. F.; Fabris, J. D.; Goulart, A. T.; Santana, G. P.; Hyperfine Interact. 1998, 3, 325.

3. Pinto, M. C. F.; Jesus Filho, M. F.; Goulart, A. T.; Fabris, J. D.; Santana, G. P.; Hyperfine Interact. 1997, 2, 61.

4. Silva, F. D.; Couceiro, P. R. C.; Fabris, J. D.; Goulart, A. T.; Ker, J. C.; Rev. Bras. Ci. Solo 2005, 29, 763.

5. da Silva, F. D.; Couceiro, P. R. da C.; Fabris, J. D.; Goulart, A. T.; Ker, J. C.; Quim. Nova 2005, 28, 5 .

6. Santana, G. P.; Fabris, J. D.; Goulart, A. T.; Rev. Bras. Ci. Solo 2001, 25, 25 .

7. Doriguetto, A. C.; Fernandes, N. G.; Persiano, A. I. C.; Nunes Filho, E.; Grenèche, J. M.; Fabris, J. D.; Phys. Chem. Miner. 2003, 30, 249.

8. SchiKorr, G.; Z. Elektrichem. 1929, 35, 65.
9. Regazzoni, A. E.; Urrutia, G. A.; Blesa, M. A.; Maroto, A. J. G.; J. Inorg. Nucl. Chem. 1981, 43, 1489.

10. Blesa, M. A.; Mijalchik, M.; Villegas, M.; React. Solid 1986, 2, 85.

11. Pinheiro, E. A.; Abreu Filho, P. P.; Galembeck, F.; Silva, E. C.; Vargas, H.; Langmuir 1987, 3, 445.

12. Coey, J. M. D. Em Iron in Soils and Clay Minerals; Stucki, J. W.; Goodmann, B. A.; Schwertmann, U., eds.; Dordrecht: Reidel, 1988.

13. Sidhu, P. S.; Gilkes, R. J.; Posner, A. M.; J. Inorg. Nucl. Chem. 1977, 39, 1953.

14. Sidhu, P. S.; Gilkes, R. J.; Posner, A. M.; J. Inorg. Nucl. Chem. 1978, 39, 1953.

15. Colombo, U.; Fagherazzi, G.; Gazzarrine, F.; Lanzavecchia, G.; Sironi, G.; Nature 1964, 202, 175.

16. Seoa, H.; Ogatac, M.; Fukuyama, H.; Phys. B 2003, 329, 932

17. Mott, N. F.; Philos. Mag. B 1980, 42, 325.

18. MØrup, S.; Paramagnetic and Superparamagnetic Relaxation Phenomena Studied by Mössbauer Spectroscopy, Polyteknisk: Forlag, 1981.

19. Whiston, C.; X-Ray Methods: analytical chemistry by open learning, John Wiley \& Sons: Chichester, 1987.

20. Aono, H.; Hirazawa, H.; Naohara, T.; Maehara, T.; Kikkawa, H.; Watanabe, Y.; Mater. Res. Bull. 2005, 40, 1126.

21. Madsen, M. B.; MÆrup, S.; Knudsen, J. M.; Hyperfine Interact. 1989, 50, 659.

22. Mitra, S.; Applied Mössbauer Spectroscopy: theory and practice for chemists and archeologists, Pergamon: New York, 1992.

23. Ferreira, S. A. D.; Santana, D. P.; Fabris, J. D.; Curi, N.; Nunes Filho, E.; Coey, J. M. D.; R. Bras. Ci. Solo 1994, 18, 167. 